\title{
Hugo y Andrade
}

$\mathbf{E}^{\mathrm{L} \text { análisis de las semejanzas estilísticas y conceptuales entre los }}$ poetas Hugo y Andrade constituye, sin duda, un interesante y absorbente estudio para el aficionado a la literatura comparada. $Y$ lo que hace atrayente desde un principio este estudio, es la polémica que existe entre los críticos literarios, algunos de los cuales mantienen que el escritor argentino no era sino un satélite en la órbita de la influencia universal de Hugo, y otros niegan rotundamente que existe la menor traza de influencia de éste sobre aquél. La polémica ha sido más bien entre críticos hispanoamericanos y españoles, pues los franceses no se han interesado en el tema.

Entre los españoles, Menéndez y Pelayo y Juan Valera han tomado parte prominente en el debate, sosteniendo, en efecto, que cualquier genio poético original que tuviese Andrade se vió disminuido por su completa subordinación al modelo francés. 1 En cambio, los críticos americanos, entre los cuales se destaca Arturo Vázquez Cey, defienden vigorosamente a sũ compatriota, reduciendo a su más mínima expresión la influencia de Hugo. 2 Y el crítico Santiago Esstrada acusa a Andrade de plagiario descarado. 3

No hemos de ocuparnos en el presente ensayo de explicar el porqué de estos puntos de vista tan distintos. Es fácil darse cuenta de que al evaluar la obra literaria de Andrade es difícil mantener completa objetividad, si se considera la exaltación patriótica con que el poeta proclama su "americanismo", concepto, sin duda, resentido por los críticos españoles por la repudiación, implícita en él, de cualquier herencia española. $Y$ no está de más imaginar que estos críticos españoles, heridos en su orgullo, se vengaran, consciente o inconscientemente, relegando al poeta Andrade al puesto poco glo- 
rioso de "satélite". Los críticos hispanoamericanos, en cambio y por la misma razón, hablan muy favorablemente del poeta argentino y defienden su originalidad.

Sea lo que sea, nadie puede negar que Andrade tenía gran admiración por el poeta francés, como de ello dan prueba la traducción de dos poemas de Hugo y la adaptación de otro, 4 la dedicatoria de una de sus mejores poesías al poeta francés, poesia que Andrade titula "A Víctor Hugo", y finalmente, en gesto de devoción al héroe, la interpolación de "Víctor" en su propio nombre. Se dice que en su conversación el nombre de Hugo pasaba con frecuencia por sus labios.

Pero es de extrañar -en vista de los numerosos comentarios sobre el tema- que sólo Santiago Estrada haya tratado de hacer un estudio comparativo de los escritos de ambos poetas, pues los demás se contentan con observaciones generales. Nosotros hemos de ocuparnos en este ensayo de diversos escritos de los dos poetas, comparándolos en cuanto al lenguaje poético, a la ideologia y a los conceptos. Al hacer tales comparaciones puede uno darse cuenta más exacta de la verdadera influencia que ejerció Hugo sobre el poeta argentino.

La semejanza de lenguaje existe principalmente vinculada con las semejanzas poéticas y conceptuales, ya que las palabras forman la trama y urdimbre de cualquier retórica. Pero vale la pena mencionar dos ejemplos aislados de comparación de vocablos. Ambos poetas emplean "negro" en uso constante y connotativo, como puede verse por estos ejemplos tomados al azar de la poesia de Andrade: negro padecer, negros recuerdos, negra servidumbre, negra y lastimera tropa; y de la poesía de Hugo: le noir janvier, le noir vieillard: décembre, de noirs éclairs, noirs soleils, l'infini, route noire, etc. $\mathrm{Y}$ resalta más aún la predilección de ambos poetas por las nubes como vehículo de expresión metafórica. Así, en Andrade tenemos: las nubes son las aves de rapiña que bajan turbulentas a devorar su carne; el cóndor anda cabalgando las nubes y viento, o cabalgando mublados y huracanes; mubes van pasando calladas; la nube voladora arrastrando sti tuinica de grana; y la nube bermeja; que recibió en la faz ruborizada el ósculo del sol en el ocaso; y en Hugo: un nuage farouche arriva; le vol éternel des nuées; la nuée, surprise; locéan est hydre et. le nuage oiseau; des nuages roulant en groupes. 
convulsifs, allumer au couchant un braiser de nuées, y la sombre nuit bâtir un porche de muées, etc.

De mayor significación son, sin embargo, los trozos que hemos de comparar en seguida. En el primer grupo la semejanza metafórica parece ser más que mera coincidencia. Andrade, a grandes y. atrevidos rasgos, traza este cuadro del iracundo y vengativo Jove que convierte los corceles en montañas:

$$
\begin{aligned}
& \text { Jove, vengativo. } \\
& \text { convirtió los corceles de granito } \\
& \text { en salvajes e inmóviles montañas! } \\
& \text { El Cáucaso caballo de batalla } \\
& \text { de algún títán caído... }
\end{aligned}
$$

("Prometeo")

Hugo, al describir la transformación del sátiro en su poema "Le Satyr" (Le Légende des Siècles), escribe: -

Ses deux cornes semblaient le Caucase et l'Atlas:

Les foudres l'entouraient avec de sourds éclats;

Sur ses flanes palpitaient des prés et des campagnes,

Et ses difformités s'étaient faites montagnes...

Tal vez sea apropiado anotar la proximidad física, en los versos anteriores, de la palabra "Caucase" a la figura gramatical "volviéndose en montañas", que nos recuerda los corceles de Andrade convirtiéndose en montañas, una de las cuales se volvió el Cáucaso.

Asimismo encontramos una mezcla de palabras y metáforas en los siguientes ejemplos. El concepto de la humanidad que se levanta de la oscuridad del mal hacia la luz de la bondad y la verdad en inevitable evolución lo expresa asi Andrade:

Tarda amanecer, pero al fin llega.

oh mal! no eres eterno!

Así como en la noche de la tierra,

profunda noche de aterido invierno,

el mundo despertó cuando en las ramas

de la selva dormida

el primer himno resonó del ave

que desplegaba el ala entumecida 
Y Hugo, después de lamentar la huranía del hombre, también siente que la fe y la esperanza hàn de recibir su premio:

N'importe, ayons foi! Tout s'agite.

Comme at fond d'un songe effrayant,

Tout marche et court, et l'homme quitte

L'ancien rive âpre et fuyant.

On va de la nuit à t'aurore

Du noir sépulcte au nid sonore.

Aparte de la idea expresada, es interesante anotar la semejanza metafórica y hasta verbal entre las frases como presintiendo a la aurora y on va de nuit à l'aurore; el primer himno resonó del ave y an nid sonore. Pero de mayor importancia, como razón comparativa, es el hecho de que el trozo de Andrade citado arriba sigue directamente al que expresa la idea de la lenta evolución histórica del hombre - y la estrofa arriba citada de Hugo la trae después de un poema que trata de esta misma idea.' Esta relación de espacio puede o no ser casual. (Para la comparación de la idea, en la forma expresada por los dos poetas, véase lo que sigue).

En sus disposiciones poéticas - los matices de sonidos que ellos oyen y de vistas que ellos ven- hay cierta comunidad de espiritu entre Hugo y Andrade. Veámoslo: al evocar los sonidos apagados, sinfónicos y susurrantes de la Naturaleza, Andrade traduce en palabras el despertar primitivo del alma:

Al eco de la insólita armonía

la tierra despertó. - La selva oscara

con ansia de volar, batió las ramas:

misteriosa y extraña vocería

se alzó del mar en la siniestra hondura

cual si ensayasen sus salvajes himnos

la borrasca y la tromba asoladora,

$y$ de la informe larva del abismo,

mariposa de luz. surgió la aurora!

("A Victor Hugo")

Menos vagas, pero evocativas de la misma atmósfera y ambiente, son estas estrofas de Hugo, tomadas de "Ecclaircie" (Les Contemplations, II) : 
Et l'âme a chaud. On sent que le nid est convé. L'infini semble plein d'un frisson de feuillée.

On croit être à cette heure où la terre eveillée

Entend le bruit que fait l'ouverture du jour.

En contraste con las atmósferas creadas por los anteriores, la próxima comparación es de interés por la semejanza de los pasajes en que ambos poetas pintan la naturaleza en sus momentos más nefastos y repugnantes. Debe observarse el uso en ambos trozos del símil, trivial como es, del viento aullando como un perro. Dice Andrade:

La negra selva por doquier! el viento como inquieto lebrel encadenado aullando en la espesural

La noche eterna por doquier! el cielo como un mar congelado, y el mar como una inmensa sepultura.

$\mathrm{Y}$ Hugo, en "Cette nuit, il pleuvait, la marée était haute" (Les Châtiments):

Les brisants aboyaient comme des chiens, le flot

Aux pleurs du ciel profond joignait son noir sanglot,

L'infini secouait et mêlait dans son urne

Les sombres tournoiments de l'abime nocturne;

Les bouches de la nuit semblaient rugir dans l'air.

De "E1 nido de cóndores", de Andrade, un trozo de interés para nuestro estudio poético comparativo es la siguiente estrofa en que describe un "peñasco sombrio":

\author{
Blanca venda de nieve lo circunda \\ de nieve que gotea \\ como la negra sangre de una herida \\ abierta en la pelea.
}

Santiago Estrada critica con razón la discordancia de este símil en que la nieve gotea como la sangre. Es de interés para nosotros, porque esta combinación de nieve y sangre es el recurso dramático usado por Hugo en su poema "Le Parricide" (La Légende. des Siecles), en el cual el espíritu de Kanut, no pudiendo descansar en 
su tumba, anda sin rumbo en busca de Dios, cubierto por un velo blanco de nieve como mortaja. Una gota de sangre, símbolo del castigo de Dios por el parricidio, salpica la blancura de la sábana:

Une goutte de sang se détachait de l'ombre,

Implacable, et tombait sur cette blancheur sombre.

Il voyait, plus tremblant qu'au vent le peuplier,

Ces taches s'élargir et se multiplier;

Une autre, une autte, une autre, une autre, o cieax funèbres!

Pero de interés aún más concreto es la frase de Hugo "livide voile", usada en otra estrofa del mismo poema, para describir el velo manchado de sangre - frase que aparece en forma idéntica en Andrade:

$Y$ hollando del Andes la frente de hielo que cubre la niebla cual cárdeno velo.

Como muchos escritores románticos, Hugo y Andrade invocaron el simbolismo majestuoso del águila volando serena en las alturas. Asi, pues, ambos poetas establecen un vínculo fraternal entre el pájaro y el poeta; Andrade cantando-

Ave de esa alborada es el poeta,

hermano de las aguilas del Cáucaso.

("Prometeo")

-y Hugo, "sublimidad desmelenada" en "Pan" (Les Feuilles d'Automne):

O poetes sacrées, échevelés sublimes

Frètes de l'aigle, aimez la montagne sauvage!

¿Puede considerarse cosa de afinidad espiritual el que ambos poetas. hablen de las águilas (es decir, los poetas) en concierto con montañas, Andrade refiriéndose al Cáucaso y Hugo a "la montagne sauvage"? Tal vez.

Pero el cóndor andino simboliza todavía mejor la hauteur romántica del tema del águila, para Andrade. $Y$ tenemos pues esta 
descripción dramática del nido del cóndor, colgando arriesgado en lo abrupto de la montaña:

Es un nido de cóndores colgado

de su cuello gigante,

que al viento de las cumbres balancea

como un pendón flotante.

Es un nido de cóndores andinos.

en cuyo negro seno

parece que fermentan las borrascas,

y que dormita el trueno!

("El nido de cóndores")

Los versos anteriores tienen un parecido con la descripción que hace Hugo de un nido de águilas, nido que también se balancea en una grandeza peligrosa:

Son nid n'est pas un nid de mousse: c'est un aire, Quelque rocher, creusé par un coup de tonnerre. Quelque brèche d'un pic, epouvantable aux yeux, Quelque croulant asile, aux flanes des monts sublimes. Qu'on voit, battu des vents, pendre entre deux abîmes,

Le noir precipice et les cieux!

$$
\text { ("A Mon Ami S. B.", Odes et Ballades) }
$$

Debido a su propio temperamento, Andrade fué atraido por el altisonante, fatídico, olimpico Hugo, de manera que su sentimentalismo bastante férvido encontró en aquél un discípulo natural. Pero aun más importante en la afinidad entre los poetas es la susceptibilidad de Andrade a las doctrinas filosóficas postuladas por el bardo francés. Notamos aquí que, en efecto, hay cierta diferencia entre el intelectualismo de Andrade y el de Hügo, diferencia de dimensión: mientras que la fuente real de la filosofía de Andrade se halla esencialmente en su amor a la patria, la de Hugo abarca a toda la humanidad. Sin embargo, muchos de los versos del argentino parecen haber salido directamente de la matriz hugónica, y tienen que ver con las exigencias del hombre universal. Tenemos aquí unos ejemplos. 
En "Prometeo", 5 adaptación de la leyenda de Prometeo, Andrade presenta al titán como el símbolo noble del "espíritu humano, soberano del mundo, verdadero emancipador de las sociedades esclávas de tiranias y supersticiones". Como tal, Promèteo es inextinguible, fértil, creador:

Todo a su paso vive, alienta, brota:
el mat, el monte, la desierta esfera;
y a su soplo creador todo se expande,
palpita y reverbera.
Levanta el polo mudo
como un arco triunfal para que pase.
sus montañas de bielo,
y enciende presuroso
sus gigantescas lámparas el Ande
para alumbrarle el tränsito del cielol

Aunque este mismo concepto se repite a menudo en la obra de Hugo, es interesante comparar el siguiente trozo, tomado de "Les Mages" (Les Contemplations, II), con los versos anteriores de Andrade. Dice Hugo, rindiendo pleitesía a "les mages", es decir, a las grandes mentalidades de todos los tiempos:

Les vents, les fiot, les cris sauonges,

L'azut, l'horreur du bois janni,

Sont les formidables breuvages

Des ces altérés d'infini:

Ils ajoutent, reveurs austères,

A leur âne tous les mystères,

Toute la matière à leurs sens:

Ils s'enivtent de l'entendue;

L'ombre est une coupe tendue

Où boivent ces sombres passants.

En estos mismos poemas otro concepto, el de la marcha inexorable de la ciencia, revelando los misterios del pasado y traspasando el umbral de un futuro sin límites, aparece expresado. Escribió Andrade:

Las venebrosas puertas del pasado rechinan a su empuje omnipotente, y se alzan en tropel a sn presencia 
desde el fondo del caos petrificado, las formas y las razas extinguidas en cuya adusta frente. el ojo de la ciencia deletrea el verdadero Génesis del mundo. que la leyenda biblica falseal

Y Hugo, dirigiéndose otra vez a los magos:

Vous voyez, fils de la nature, Apparaître à votre flambear

Des faces de lumière pure, Larves du vrai, spectres du beau; Le mystère, en Grèce, en Chaldée Penseurs, grave a vos front l'idée

Et l'hieroglyphe à vos murs

Et les Indes at les Egyptes

Dans les ténèbres de vos cryptes.

S'enforcent en porches obscars!

Tal vez la unión más perfecta entre Hugo y Andrade la encontramos en la preocupación de ambos por el futuro: su visión de paz y amor entre los hombres, de progreso hacia la realización de los ideales de una sociedad democrática. Como se ha dicho, el alcance del sueño de Andrade no abarcó tanto, a veces, como el horizonte universal contemplado por Hugo. Las estrofas comparadas más abajo dan prueba de esta diferencia de visión, pero también muestran el fervor inspirado con que ambos poetas cantan el futuro. Andrade honra a la patria; Hugo a todas las naciones. La exaltación es la misma:

De pie para cantarla! que es la patria, Ja patria bendecida, siempre en pos de sublimes ideales, el pueblo joven que arralló en la cuna el rumor de los himnos inmortales! $Y$ que boy llama al festín de su opulencia a cuantos rinden culto a la sagrada libertad, hermana del arte, del progreso y de la ciencia,¡la patrial que ensanchó sus horizontes rompiendo las barreras 
que en otrora su espiritu aterraton, y a cuyo paso en los nevados montes del Génesis los ecos despertaron!

¡La patria! en ella cabe cuanto de grande el pensamiento alcanzá, en ella el sol de redención se enciende, ella al encuentro del faturo avanza, y a su mano, del Plata desbordante la inmensa copa a las naciones tiende!

("Atlántida")

Oui, je vous le déclare, oui, je vous le repète,

Car le clairon redit ce que dit la trompette,

Tout sera paix et jour!

Liberté! plus de serf et plus de proletaire!

O sourire d'en haut! o du ciel pour la terre

Majestueux amour!

L'arbre saint du Progrès, autrefois chimérique,

Croitra, couvrant l'Europe et couvrant l'Amérique,

Sur le passé détruit,

Et, laissant l'etber pur luire à travers ses branches,

Le jour, apparaitra plein de colombes blanches,

Plein d'etoiles, la nuit.

Esta misma intensidad acerba y casi estridente, continúa en los siguientes versos de utópica visión: rotas las cadenas del pasado, terminada la asfixia de tres siglos (España), la nave del Estado se lanza a su gloriosa vanguardia:

IEs mi patria! mi patria! Yo la veo a vanguardia de un mundo redimido, de un muado por tres siglos amarrado, que cual bajel en mar desconocido rompiendo las cadenas del pasado, se lanza con andacia. cargado de celestes esperanzas, al puerto de la santa democracia! Es su bandera aquella que flamea en las rocas del Cabo seculares, la que lleva a una raza esclavizada la luz de libertad de sus altares; 
la que preside el colosal concierto

de la conciencia humana emancipada

mientras rueda a sus pies el tronco yerto

del fanatismo vil, que en hora impia

la mantuvo en sus brazos sofocada!

Y así también declara Hugo su promesa fatídica. Anotemos, de paso, que el simbolismo de Andrade está sintetizado en un "bajel... rompiendo las cadenas del pasado" y, a manera de comparación, veamos cómo, en los versos siguientes, el motivo simbólico de Hugo en su poema "Plein Ciel" (La Légende des Siècles) es una nave que rompe sus amarras con un pasado terrestre. Pero no debemos olvidar, al comparar los versos de ambos poetas, la diferencia en cuanto al alcance de la visión:

\section{Oh! ce fut tout à coup}

Comme une éruption de folie et de joie

Quand, près six mille ans dans la fatale voie

Défaite brusquement par l'invisible mair,

Le pesanteur, liée au pied du genre humain,

Se brisa; cette chaîne était toutes les chaînes!

Tout s'envola dans l'homme, et les fureurs, les haines,

Lies chimères, la farce évanouie enfin.

L'ignorance et l'erreur, la misère et la faim.

Le droit divin des rois, les faux dieux juifs on guèbres

Le mënsonge, le dol, les brumes, les ténèbres.

Tomberent dans la poudre avec l'antique sort.

Comme le vêtement du bagne dont on sort.

No es muy aventurado pensar que Andrade, con gran dolor; leyó cuidadosamente el tomo de versos L'Anée Terrible, en el cual describe Hugo aquel año funesto de $1870-71$ en que los alemanes sitiaron y tomaron a París, ciudad donde se hallaba Hugo que habia regresado de su destierro. En el poema de Andrade "A Víctor Hugo" se nota fácilmente la influencia de L'Anće Terrible. 6 Los versos siguientes

¿Qué lentas son las horas de la historia!

Qué largo y qué sombrio

el imperio del mal! 
no sè diferencian mucho de éstos de Hugo, que expresan esencialmente la misma idea, tomados del poema "Loi de Formation du Progrés":

Oht dans l'ascension humaine, que la marche

Est lente, et comme on sent la pesantenr de l'arche!

Refiriéndose otra vez a la historia -es decir, al curso de los sucesos humanos-, es pertinente anotar aquí las observaciones de Arturo Vázquez Cey, quien indica que al concebir el movimiento histórico como una lucha tenaz entre la verdad y el error, entre el fanatismo y el progreso, Andrade fué discípulo de Michelet, y cita como prueba de la influencia de éste los dos primeros versos de la segunda estrofa que vemos en seguida:

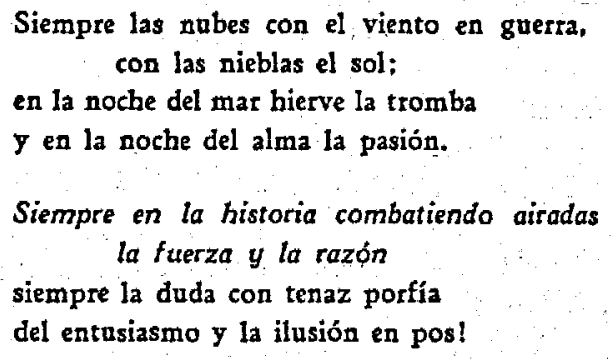

Esta afinidad con Michelet es legítima. Pero también tenemos ejemplos de tal tesis en versos de Hugo, y hasta puede decirse que nada le era tan caro a Hugo como el principio de las fuerzas opuestas, que no es sino otra manera de expresar la ley de, antítesis, su más grande obsesión en la técnica. Los versos siguientes son representativos de su manera de expresar este tema:

Donc, la matière prend à l'idéal, et tire L'esprit vers l'animal. l'ange vers le satyre, Le sommet vers le bas, l'amour vers l'appétit, Avec le grand qui croule elle fait le petit.

$Y$ aunque las "horas de la historia" han marchado lentamente; ambos poetas ven en el siglo XIX la culminación de muchos de los infortunios de la humanidad $y$, en los versos que vamos a comparar, 
proclaman la llegada de un grande y nuevo ímpetu. Acerca de esta era de fertilidad y de inquietud escribió Andrade:

\author{
La aurora de este siglo \\ nació en los tenebrosos horizontes \\ de un inmenso desierto. \\ Tribus errantes $y$ salvajes montes, \\ la barbarie doquiér; $y$ el fanatismo \\ fué ascendiendo, ascendiendo, \\ como tin rayo de luz en un abismo, \\ y al bajar al ocaso, \\ alumbran su camino \\ los millares de antorchas del progreso, \\ del pensamiento al resplandor divino!
}

("San Martin")

Versificó Hugo en "Les Deux Trophées" (L'Année Terrible) acerca 'de este mismo siglo y de su rica cosecha:

Peuple, ce siècle a va tes travaux surbumains.

Il t'a va repétrir l'Europe dans tes mains.

Tu montras le néant du sceptre et des couronnes

Par ta façon de faire et défaire des trones:

A chacun de tes pas tout croissait d'un degré;

Tu marchais, tu faisais sur le globe effaté

Un ensemencement formidable d'idées. . .

Tu détruisais le mal, l'enfer, l'erreur, le vice,

Ici le moyen âge et là le saint-office:

Superbe, th luttais contre tout ce qui nnit;

$T a$ clarte grandissante engloutissait la nuit;

Tandis que tu montais dans ta voie etoilée...

Terminamos nuestras citas con un trozo tomado de la prosa de Andrade en que está resumida su relación espiritual con Hugo, trozo en el cual vemos no sólo un homenaje de referencia al Maestro, sino ciertas características del estilo de Hugo - el Hugo de Les Châtiments - en su incisiva cadencia, su fuerza antitética y su movimiento en crescendo: 
Victor ha dicha:

Los obstinados son los sublimes.

Las medianías se dejan disuadir por el obstáculo especioso: los fuertes, no! Parecer, es su tal vez; conquistar es : su certeza. Las pequeñas conciencias guiñan el ojo: las grandes echan relámpagos.

Perseveremos, pues, Si no podemos escribir, conspiremos. Si no podemos conspirar, maldigamos. Si no podemos maldecir, juremos en el fondo del alma, vengat uno por uno, los vejámenes de que en este momento somos victimas.

Combatamos! Sea la prensa la Némesis implacable que azote la espalda de los verdugos de la República: sea la prensa la musa airada del patriotismo que convoque a las generaciones nuevas para las grandes lides de la tedención!

\section{* * *}

En vista de nuestro estudio nos parece que la polémica de los críticos acerca de la originalidad o el plagio en las obras poéticas de Andrade no tiene razón de ser. Porque plagio significa una actitud hostil : el escritór que roba ideas, o formas de expresión, generalmente ataca abierțmente al escritor de quien las roba, o por lo menos asume contra él una actitud hostil. No hay tal actitud de hostilidad en Andrade hacia Hugo.

El caso de Andrade y Hugo, pues, puede ser considerado como una confluencia lógica y comprensible de dos mentes y dos corazones. $\mathrm{Y}$ nos parece de poca importancia que sea Andrade el responsable de esta confluencia; lo que importa es que ambos poetas fueron contemporáneos en una era en que el florecimiento del romanticismo dió cierta homogeneidad ideológica, técnica y sentimental a la textura de toda expresión estética en el mundo occidental. En su compartir de esta contemporaneidad existe mucho de la base de la afinidad de Andrade con Hugo.

De las numerosas facetas del romanticismo, la que impresionó más a Andrade fué la de sus ideales sociales - el ideal de la democracia, de la libertad personal, de la justicia, y el de la liberación de las fuerzas intelectuales y materiales que iban a alcanzar una sociedad utópica; en una palabra, del progreso humano. $Y$ así, como poeta civico, Andrade encontró fácilmente su numen en Hugo, una de las fuentes principales de la poesía humanitaria. Está claro que la musa de Andrade no se conmovió en su máxima expresión 
sino durante la tarde del siglo XIX, época en que el nombre de Hugo pasaba a su ocaso; pero no fué conmovido Andrade por el culto de ese tiempo de Verlaine con su principio de "l'art pour l'art" ni tampoco por el simbolismo complejo de Mallarmé. Más bien, Andrade volvió al próximo pasado, del cural tomó los sonidos viriles de la trompeta hugónica, y trató, con menos eficacia en la mayor parte pero con igual pasión, de transcribir su mensaje.

Debemos también recordar la vaga analogía que existía entre el ambiente politico de Andrade y el de Hugo: Francia, en vida de una generación, sufrió la grande Revolución, sintió la tiranía del imperialismo, la ineficacia del monarquismo restaurado y luego (en vida de Andrade) se esforzaba por realizar el estadó republicano; esta Francia, según Andrade, se parecía a su propia patria, que hacia poco tiempo se habia librado del dominio metropolitano, había sufrido bajo la tirania de Rosas y buscaba entonces su propia y nueva realidad.

En fin, tenemos que recordar el temperamento de Andrade: en su amor por lo grandioso y lo inmenso, en su latir con visiones del futuro casi al punto de alucinarse, en su delirio de vocablos, metáforas y figuras gramaticales, y en su predilección por el uso de la antítesis - todo lo cual vió consumado tan sublimemente en Hugo-, Andrạde adquirió la luz abrasadora y el ademán olímpico de Hugo mismo. rica.

A pesar de la pobreza de su obra, Andrade fué el Hugo de Amé-

$$
\begin{aligned}
& \text { JAMES A. Granier, } \\
& \text { The Hispanic Foundation, } \\
& \text { The Library of Congress. }
\end{aligned}
$$

NOTAS

1 Menéndez y Pelayo ha escrito: "Andrade tenía el gusto sin educar. y le fascinó la imitación de lo peor de Victor Hugo, por quien profesaba una especie de culto, a más bien de fanatismo. . En él como en Víctor Hugo. fatiga la monotonia de lo grandioso, la luz abrasadora de mediodia derramada por igual y de plano sobre todos los objetos. $Y$ como en todo imitador, aun siendo tan distinguido como Andrade, se extreman los defectos y no 
las cualidades del modelo... Andrade... introduce las más enfáticas y prosaicas vilgaridades. Verdad es que lo mismo hacía Victor Hugo... su [de Andrade] pomposo latinismo de raza... viene a resolverse en un galicismo perpetuo..." Historia de la poesia hispano-americana (Madrid, 1913). tomo II, pp. $46 \mathrm{~T}-463$.

A Juan Valera le parece inty admirable Andrade cuando éste imita" a Zorrilla, pero en cuanto a su relación con Hugo dice: "Hay también extravagancias en Andrade, asi en el fondo como en la forma, de cuyas cuartas partes, lo menos, hago yo responsable a Victor Hugo y a la mania que inspira de imitarle". Cartas americanas, Obras completas, tomo XLI. p. 138.

2 Por ejemplo: "Andrade ha sido abrumado largo tiempo con la acusación de imitador de Hugo... ¿ Existe similitud? No encontramos ninguna. ni en los temas, ni el procedimiento, ni el arte de uno. Y otro". (1) Evar Méndez, Olegatio V. Andrade, Obras poéticas ordenadas y con un prólogo de... (Buenos Aires, 1918), p. 18.

3 La critica de Estrada sobre Andrade se encuentra en sn obra Miscelánea (Barcelona, 1889), tomo I. A causa de su férvido catolicismo, Santiago Estrada fué ofendido por el leve librepensamiento de Andrade $y$ tomó ocasión de acusarle de plagiario. Pero sus ejemplos de latrocinio literario se reducen. a veces, ad absurdum, como lo vemos aquí: "Había himno en la flor y resplandor en el ruido', dice Hugo; y Andrade, refiriéndose a la frente del hombre redimido: "Hay luz y voz en ella" ", p. 150.

4 "Le Crépuscule" (Les Contemplations, I), "Stella", (Les Chätiments, II) y "Nature" (Les Contemplations, I).

5 Escribiendo del tiempo en que Andrade compuso sa "Prometeo", Váz. quez Cey dijo: "Renan y Darwin, dispensadores entonces en Buenos Aires de la sapiencia anticlerical, fueron sin duda, en esa circunstancia, númenes de Andrade. Y Hugo, aquel Hugo que específicamente en Les Mages y de suerte difusa en toda su producción celebra a los prohombres del espiritu humano, aletea en el poderoso conjunto". La poesia de Olegario $V$. Andrade y su época (Buenos Aires, 1921), p. 54.

6 En este mismo poema asi escribió Andrade en cuanto a los alemanes: "Era la Francia... la Francia de tu amor, que tambaleaba herida por el hacha germana". 